\title{
Subject Index
}

Abrasive systems, dentifrices 123

Absorption 23, 24, 38, 78

Accidental poisonings 66, 67, 69, 70, see also Acute toxicity

Acid-base status, see also $\mathrm{pH}$

metabolism effects 28,29

toxicity effects 77,78

Acidulated phosphate fluoride 71, 72, 75

Acute toxicity $66-80$

defined 78

factors influencing $75-78$

gastric effects $72-75$

probably toxic dose $67,68,71,78$

source $68,69,85,86$

dental products $67,69-72,78,79$

treatment $24,26,68,78$

Administration methods, see Delivery methods

Age factors, see also Children

dental fluorosis $85-87$

recommended intake 84

toxicity effects 76,77

Alkalosis 78

Altitude 30

Ameloblasts 89-91, 93

Amelogenins 90-93

American Academy of Pediatric Dentistry 130

American Dental Association

dietary supplement guidelines 11 ,

141,142

tablet guidelines 71

topical fluoride guidelines 130

water fluoridation statement 136

Amine fluoride 117, 160, 168

Ammonium fluoride 117

Amorphous calcium phosphate agents 152

Apatite 91-93, 98-101
Bacteria

fluoride binding model 147, 148

fluoride effects $108-110$

mineral dynamics $101,102,107$

Beikost 13

Beverages, infant 13,84

Biofilm 99, 102

Biological F binding sites 147, 148

Biomarkers

classification $38,48,53,63$

contemporary

blood plasma $39-41,48$

bone surface $27,39-42,48$

early investigations 38,39

human milk 43, 44

individual versus group 46,47

saliva $42,43,48,149,150$

sweat 43

urine $44-46,48,49$

defined 53

historical

bone 61,62

teeth $62,63,103$

recent

hair 60,61

nails $53-60$

Blood plasma

fluoride distribution 25

sample collection 39,48

Blood plasma concentration

after ingestion 22, 23

biomarker $39-41,48$

factors influencing 77,82

pharmacokinetics 25

rhythmicity 31

total 25 
Body burden 38, 41, 62

Body fluids, specialized, fluoride distribution 26

Body tissues, fluoride distribution to 25-27

Bone

fluoride metabolism 23, 26, 27, 62, 77

fluoride reservoirs $38,41,87$

sample collection $41,42,62$

types $41,42,62$

Bone biomarkers

contemporary, bone surface $27,39-42,48$

historical 61,62

Bottled water 6,136

Buffer extraction, plaque fluoride reservoirs 151

Calcified tissues, see also Mineral dynamics fluoride distribution $23,26,27,38,62$

Calcium fluoride dental erosion $160-164$

oral reservoirs 105-107, 110, 147, 149, 152

plaque reservoirs $110,147,151,153,154$

Calcium fluoride-like material 104, 147, 149, 151, 154, 155

Calcium, fluoride metabolism 24, 28, 32, 68

Calcium phosphate agents, amorphous 152

Calcium pre-rinse systems $153-155$

Calculus formation 101

Canadian Dental Association 11

Cancellous bone 41, 42, 62

Caries control

fluoride mechanisms of action 102-111, 134

oral fluoride reservoirs, see Oral fluoride reservoirs

systemic fluoride 107, 108, 136, 137

topical fluoride, see Topical fluoride

Caries development

biochemistry $98-102$

etiology 97, 98

plaque $\mathrm{F}$ release 151

Cerebrospinal fluid, fluoride distribution 26

Chemical compounds, see Compounds

Children

acute toxicity $67,69-71,76,77,79$

biomarkers

hair 61

nails 59,60

teeth 63

urine $45-48$

dental fluorosis, see Dental fluorosis fluoride intake 1-19

'optimal' 2, 83, 84

sources 4-13

renal impairment 29,30

systemic methods $84,140-142$

topical methods $124,125,130$

Child-resistant caps 70, 79

Chronic toxicity, see Dental fluorosis

Circadian rhythm 30, 31

Cochrane Central Register of Controlled Trials 116

Collagen 99, 166

Community delivery methods $115,116,143$

milk fluoridation $135,140,141$

salt fluoridation $135,138-140$

water, see Water fluoridation

Community level biomarkers 46, 47

Compact bone 41, 42, 62

Compounds, see also specific compound

dental erosion 163-168

systemic fluoride 135

topical fluoride $116,117,126,127$

toxicity effects 75,76

Concentration, see also Biomarkers

dentifrices 124-126

systemic methods $135,139,140$

toxicity effects by 73

Consumer Product Safety Commission 70

Controlled release rinses 152, 153

Copolymer membrane device 128

Cortical bone 39

Costs

professional topical methods 129

salt fluoridation 138

water fluoridation 135,136

Critical pH 101

Crystal surface fluoride 104, 105, 108

Daily urinary fluoride excretion $45,46,48$

DDE index 31

Dean, Trendley 134

Dean fluorosis index 83,84

Delivery methods $115,116,143$

community, see Community delivery methods

professional 116, 121, 122, 143, see also Topical

fluoride

self-applied 116, 143, see also Topical fluoride systemic administration, see Systemic fluoride topical administration, see Topical fluoride

Demineralization, see Mineral dynamics 
Dental erosion $158-170$

defined 158

dentin 158, 159, 166-168

enamel $159-166$

prevention 160

Dental fluorosis 81-96

children 1-19

biomarkers predicting 59-61, 63

epidemiology 85-87

nutritional status 31

renal impairment 30

window of maximum susceptibility $2-4$

clinical manifestations 82,83

etiology 82, 84, 85, 136

genetic susceptibility $32,33,82,86-88$

history 133,134

indices $31,83,84$

pathology $86-93$

prevalence $5,85,86$

prevention 84

risk factors $1,2,14$

treatment 84

Dental products, see Topical fluoride, see also specific product

Dentifrices 122-127

acute toxicity $69-72,75$

children's fluoride intake 6-10

clinical efficacy 119, 124-127, 129

composition 123, 126, 127

dental erosion 162, 163, 166

fluoride concentration $75,124-126$

low-fluoride 124, 125

pH 126

plaque fluoride reservoirs 151,152

Dentin

biomarkers 62,63

composition 98, 99

erosion $158,159,166-168$

fluoride distribution to 26,27

fluorosis 86,87

mineral dynamics $98,99,111,112$

Diet

biomarker effects 48

dental fluorosis 87

fluoride metabolism effects 24, 29, 31, 32

Dietary acids 158

Dietary reference intakes 84

Dietary supplements 141,142

children's intake $10,11,84,142$ child-resistant caps 70,79

compounds and concentrations 135,141

Disodium hexafluorosilicate 136

Disodium monofluorophosphate 24, 75, 140

Dissociated fluoride, see Ionic fluoride

Distribution 23, 25-27, 38, 62

DMFS pooled estimates 129

Drinking water fluoridation, see Water fluoridation

Drops 141

Ductal saliva 42,43

Enamel

altitude effects 30

biomarkers 62, 63, 103

composition 98, 99

dissolution 100, 101

erosion $158-166$

fluoride distribution to 26,27

fluorosis $81,82,86-93$, see also Dental fluorosis

mineral dynamics $92,93,98-100,111,112$

Enamel fluid fluoride 104-106, 108

Environmental sources 60,61

Erosion, see Dental erosion

European Academy of Paediatric Dentistry 130

Excretion

sweat 23

urinary, see Urinary excretion

Exercise 30

Exogenous sources 60

Extracellular fluids 41,78

Extraction techniques, plaque fluoride reservoirs 151

F binding sites 147,148

Fecal fluoride 23, 28

Fingernails, biomarker 53-60

Fluorapatite 146, 147, 149, 150

Fluorhydroxyapatite 101-103

Fluoride

administration methods, see Delivery methods

cariostatic benefits, see Caries control

compounds, see Compounds

excretion, see Excretion

exposure, biological markers, see Biomarkers

intake, see Ingestion

metabolism, see Metabolism

sources, see Sources, see also specific source

toxicity

acute, see Acute toxicity

chronic, see Dental fluorosis 
Fluoridens 133

Fluorosilicic acid 136

Fluorosis, see Dental fluorosis

Foams 118

acute toxicity 71,122

clinical efficacy 122

Free saliva 149,150

Gastrointestinal tract absorption $23,24,38,78$

toxicity effects $72-76$

Gels 118

acute toxicity $71,72,75,122$

application guidelines 121

clinical efficacy $119,122,129$

Gender

biomarkers 58, 59, 62

dental fluorosis 87

Genetic factors $32,33,82,86-88$

Geographical area, nails, biomarkers 58, 59

Gingival crevicular fluid fluoride levels 26

Glass beads 128

Glomerular filtration rate 27,28

Ground waters 85

Group biomarkers 46, 47

Hair biomarker 60, 61

Healthy People 2010, 135

Henderson-Hasselbalch equation 72,73

Hexafluorosilicic acid 136

Hexamethyldisiloxane-facilitated diffusion 57 , 58

Hormones 30,31

Human Development Index 137, 138

Human milk 26, 43, 44

Hydrogen fluoride

metabolism 21, 24, 28, 29

toxicity $72-75,78$

urinary excretion 45

Hydroxyapatite 91-93, 98-101, 107

Hydroxyapatite-Eudragit RS100 diffusion-controlled fluoride system 128

Hydroxyethyl methacrylate/methyl methacrylate copolymer 128

Hypertension 139

Individual biomarkers 46,47

Infant foods, manufactured 13,84

Infant formulas $6,11-13,84$
Ingestion, see also Systemic fluoride acute toxicity, see Acute toxicity plasma concentration curve 22,23

Inorganic fluoride compounds 116,117 natural occurrence 20,85

In situ de/remineralization test protocol 152, 153

Institute of Medicine, US Food and Nutrition Board 84

Intracellular concentration 78

Intraoral fluoride reservoirs, see Oral fluoride reservoirs

Ion activity product 99, 100, 147

Ionic fluoride 21, 25, 27

blood plasma 39

ground waters 85

human milk 44

renal clearance 27,28

Kallikrein 4 90, 91

Kidneys, fluoride clearance 27-31, 44, 45, 78

Lethal dose $67,68,71,78$

Lozenges 133, 141

Malnutrition 31

Matrix proteins 90-93, 99

McKay, Frederick 133

Metabolic alkalosis 78

Metabolism 20-36

absorption 23, 24

distribution 25-27

factors, modifying $28-33$

fecal clearance 28

general features 21-23

renal clearance $27-30,44,45,78$

Metal ions, polyvalent 163-165

Milk

animal

calcium content 32,68

fluoridation 135, 140, 141

human $26,43,44$

Mineral dynamics

biochemistry 99-101

caries formation 101-103

dental erosion 160, 161

dentin 98, 99, 111, 112

enamel 92, 93, 98-100, 111, 112

enhancement 107, 152, 153 
fluoride effects 92, 93, 104-107, 111, 112

saliva 99, 100, 107

Mineralized tissues, fluoride distribution 23,

$26,27,38,62$

MMP-20 90, 91

Monofluorophosphate-containing compounds 116, 117

Mottled enamel, see Dental fluorosis

Mousses 118

Mouthwashes, see Rinses

Mucosa

fluoride reservoirs 110, 149, 150

toxicity effects $73-76$

Nails, biomarker 53-60

National Brazilian Epidemiologic Survey 137

National Research Council, Committee of Biomarkers 53

Natural sources 20, 85

Non-ionic fluoride 25, 39

Nutritional status 31, 87, see also Diet

'Optimal' fluoride intake 2, 83

Oral bacteria, see Bacteria

Oral fluoride reservoirs caries control 105-108, 110, 111

enhancement $152-155$

location 148-152

types $104,105,146-148$

Organic compounds 116, 117

Outer fluoride 104

Overdose, see Acute toxicity

Parathormone 30

Perikymata 82

$\mathrm{pH}$

critical 101

dental erosion $160,166,167$

dentifrice 126

enamel formation 92

fluoride metabolism 21, 24-31, 45, 78

fluoride toxicity $73-75,77,78,92$

mineral dynamics 99-103, 107, 111

Pharmacokinetics 25, 62

Phosphatases 75, 76

Physical activity 30

Plaque

fluid microenvironment 99, 100

fluoride concentration 125,150 fluoride reservoirs $108,110,111,147,150-155$

Plasma, see Blood plasma

Poisonings, accidental 66, 67, 69, 70, see also Acute toxicity

Potassium fluoride 139

Pre-rinse systems, calcium 153-155

Probably toxic dose $67,68,71,78$

Professional delivery methods 116, 121, 122, 143, see also Topical fluoride

Proteinases 90, 91

Proteolytic activity $90-92$

Reference dose 84

Remineralization, see Mineral dynamics

Renal clearance 27-31, 44, 45, 78

Rice-based diet 32

Rinses 122

acute toxicity $69-72,79$

calcium pre-rinse systems $153-155$

clinical efficacy 120, 129

controlled release 152,153

oral fluoride reservoirs $152-155$

plaque fluoride reservoirs 151, 152

tin-containing 166-168

Saliva

biomarkers 42, 43, 48, 149, 150

fluoride distribution 26

fluoride reservoirs $147,149-155$

mineral dynamics 99, 100, 107

slow-release fluoride devices 128

Salmon thyrocalcitonin 30

Salt fluoridation $135,138-140$

Self-applied methods 116, 143, see also Topical fluoride

Silane fluoride 117

Skeleton, see Bone

Slow-release fluoride devices 128

Sodium fluoride

absorption 24

acute toxicity $66,67,71,75$

controlled release systems 153

dental erosion 160, 163, 166, 168

dietary supplements 141

milk fluoridation 140

rinses 153,154

salt fluoridation 139

topical formulations $117,126,127$

Sodium fluorosilicate 136 
Sodium lauryl sulfate 151,155

Sodium monofluorophosphate $117,126,127$

Soft tissues, fluoride distribution to 23, 25, 26

Solid phase fluoride 104, 108

Solubility product 99,147

Solutions 72, 116-118

Sorghum-based diet 32

Sources

children's intake 4-13

dental products, see Topical fluoride, specific product

dietary, see Dietary supplements

environmental 60,61

infant foods/beverages 13,84

infant formulas $6,11-13,84$

milk 135, 140, 141

salt $135,138-140$

toxicity $68,69,78,79,85,86$

water, see Water fluoridation

Stannous fluoride 117, 163

Steady-state distribution 25, 41

Stomach

absorption $23,24,78$

toxicity effects $72-76$

Stomach acids 158

Striae of Retzius 82

Sucrose 140

Sweat 23, 43

Systemic fluoride $133-145$

acute toxicity, see Acute toxicity

caries control 107, 108, 136, 137

compounds 135

sources, see Sources

Tablets 71,141

Tamarind 32

Tartaric acid 32

Therapeutic ratio 38,48

Thylstrup and Fejerskov Fluorosis Index 83, 84

Tin-containing fluoride products $165-168$

Titanium tetrafluoride 117, 163-165, 167

Toenails, biomarker 53-60

Tooth

biomarkers 62,63

caries control, see Caries control

enamel, see Enamel

erosion, see Dental erosion

fluoride reservoirs 148,149

fluorosis, see Dental fluorosis
Toothbrushing habits $127,162,163$

Tooth formation

fluoride effects 2-4, 88-92

mineralization, see Mineral dynamics

renal impairment effects 29,30

Toothpaste, see Dentifrices

Topical fluoride 115-132

acute toxicity $67,69-72,78,79$

child-resistant caps 70,79

chronic toxicity 85,86 , see also Dental fluorosis

combination modalities 128,129

compounds 116, 117, 126, 127

defined 116

dentifrices, see Dentifrices

gels, see Gels

mechanisms of action 103-111, 134

mousses 118

reservoirs, see Oral fluoride reservoirs

rinses, see Rinses

slow-release devices 128

solutions $116-118$

varnishes, see Varnishes

Total daily fluoride intake $45,46,48$

Total plasma fluoride 25

Toxicity

acute, see Acute toxicity

chronic, see Dental fluorosis

Trabecular bone 39

Underground water 85

Urinary excretion

biomarker 44-46, 48, 49

range of values $45,47,48$

factors affecting $31,32,44,45,48$

physiology 27, 28, 38

US Centers for Disease Control and Prevention 136

US Environmental Protection Agency 84

US Food and Drug Administration 153

Varnishes 120

acute toxicity 122

application guidelines 121

clinical efficacy $119,122,129$

for dental erosion 162,163

Vegetarian diet 29, 31, 32, 48

Water

bottled 6,136

underground 85 
Water extraction, plaque fluoride reservoirs 151

Water fluoridation $135-138$

acute toxicity 68,69

children's intake 4-6

chronic toxicity $82,85,86,136$

compounds and concentrations 135,136

costs 135,136

history 134

mechanism of action 107, 108, 134 nails, biomarker 58

opposition 136, 138

Water rinsing, plaque fluoride effects 150,151

Whole saliva $42,43,149,150$

World Health Organization

biomarker classification 53

biomarker statement 37,47

salt fluoridation statement 139

water fluoridation statement 136 